\title{
Filipino Midwives' Knowledge, Self-perceived Role and Experiences in Educating Parents of Families with Newborns who are Confirmed Cases of Glucose 6 Phosphate Dehydrogenase Deficiency
}

\author{
Romer J. Guerbo, ${ }^{1}$ Carmencita D. Padilla, ${ }^{2,3}$ Mercy Y. Laurino, ${ }^{2,4}$ Ellen S. Regalado, ${ }^{5}$ \\ Catherine Lynn T. Silao ${ }^{3,6}$ and Ernesto R. Gregorio, Jr. ${ }^{7}$ \\ ${ }^{1}$ Nerwborn Screening Center Mindanao, Southern Philippines Medical Center, Davao City \\ ${ }^{2}$ Department of Pediatrics, College of Medicine and Philippine General Hospital, University of the Philippines Manila \\ ${ }^{3}$ Institute of Human Genetics, National Institutes of Health, University of the Philippines Manila \\ ${ }^{4}$ Cancer Prevention Programs, Seattle Cancer Care Alliance \\ ${ }^{5}$ University of Texas Health Science Center \\ ${ }^{6}$ College of Medicine, University of the Philippines Manila \\ College of Public Health, University of the Philippines Manila
}

\begin{abstract}
Introduction. Midwives play an important role in promoting newborn screening (NBS) and they ensure that all Filipino newborns are offered screening for life-threatening metabolic conditions. Of the disorders included in NBS, Glucose 6 Phosphate Dehydrogenase (G6PD) deficiency is the most common disorder detected.
\end{abstract}

Objectives. This study aimed to assess the knowledge, self-perceived role, and experience of midwives who practice in urban and rural settings in educating parents of a newborn who are confirmed cases for G6PD deficiency.

Method. One-on-one semi structured interview was conducted among 21 midwives from Manila City and Lipa, Batangas, Philippines.

Results. The study findings indicate that midwives frequently serve as the primary information resource for parents of infants with G6PD deficiency. Assessment of knowledge showed that midwives have sufficient knowledge about the medical management and the necessary follow-up of infants with G6PD deficiency. However, it also revealed that they have inadequate knowledge of the underlying genetic cause of G6PD deficiency. The surveyed midwives recognized their role and the importance of proper education regarding G6PD deficiency.

Conclusion. The findings of this study identified gaps in the midwives' knowledge on the genetic mechanisms and inheritance of G6PD deficiency, which could be a basis to improve the education and dissemination of information and to eventually improve parental education and care of newborns with G6PD deficiency.

Key Words: Genetic counseling, Glucosephosphate Dehydrogenase Deficiency, Neonatal Screening

\section{INTRODUCTION}

Paper presented as poster at the $13^{\text {th }}$ Asia Pacific Conference on Human Genetics, November 7-9, 2019, Makati Shangri-La, Manila, Philippines and at the $12^{\text {th }}$ Asia Pacific Conference on Human Genetics, November 8-10, 2017, Royal Orchid Sheraton Hotel, Bangkok, Thailand.

Corresponding author: Romer J. Guerbo, RN, MSc

Newborn Screening Center Mindanao

Southern Philippines Medical Center

J.P. Laurel Avenue, Bajada, Davao City 8000, Philippines

Email: rguerbo.nscm@gmail.com
Glucose 6 Phosphate Dehydrogenase (G6PD) deficiency is the most common human enzyme defect. It affects more than 400 million people worldwide, and in the Philippines, it has a prevalence of one out of 56 newborn babies. ${ }^{1}$ The most frequent clinical manifestations of G6PD deficiency are neonatal jaundice and acute hemolytic anemia, which are usually triggered by certain foods and drugs. Numerous drugs and chemicals, such as primaquine, dapsone, and ingestion of fava beans can induce hemolytic anemia in G6PD-deficient individuals. ${ }^{2}$ G6PD deficiency may cause 
excessive hyperbilirubinemia leading to kernicterus, one main cause of mental retardation, or even death among newborns affected with the condition. Thus, if a newborn is found to be G6PD-deficient, counseling and adequate education for parents are necessary. ${ }^{3,4,5}$ Physicians, nurses, and midwives ensure that newborn screening (NBS) is provided to all Filipino newborn babies. Midwives are at the frontlines of the Philippine health care system and are sometimes the only resource person in rural areas, community settings, and also in some urban areas. They perform unique tasks which include but are not limited to: supervision and care of women during pregnancy, delivery and health education of the patient, family, and the community in the aspects of nutrition, reproductive health, immunization, health education, and counseling. They play an important role in promoting NBS in hospitals and in the community setting and are expected to ensure that NBS services such as information, education, and communication are provided for each parent. ${ }^{6}$

Genetic counseling is an important element of medical genetics. The process helps parents better understand the condition, hopefully bringing positive management outcome and prevention of future complications. Because of the need for genetic counseling in the Philippines, the master's program in genetic counseling was established in 2011 at the University of the Philippines Manila to increase the number of professionals who will provide counseling to families. ${ }^{7}$ As part of the healthcare team, midwives and other allied healthcare providers must understand basic information of conditions included in the newborn screening panel since they are, oftentimes, the first contact of care for families. Given the limited number of genetic counseling professionals that can provide genetic counseling services, this study would also want to explore the possibility of our midwives as counterpart of genetic counselors in the community setting. However, there is limited data on the midwifes' knowledge, experience and self-perceived role in the NBS program. This information is important in identifying the needs and future programs to improve delivery of NBS and genetic counseling services in the country.

This study aimed to describe the knowledge, selfperceived role, and experiences of midwives in educating parents of children who are confirmed cases for G6PD deficiency.

\section{METHODS}

\section{Study Design and Population}

The study had a descriptive cross-sectional approach that utilized a purposive sampling technique. The study participants were Filipino midwives from Manila (urban setting) and Lipa, Batangas (rural setting), who provided care to parents with children who are confirmed cases for G6PD deficiency. The study was approved by the UP-Manila Research Ethics Board (UPMREB) last June 30, 2015 with a protocol code UPMREB 2015-208-01.

\section{Procedure}

Invitation letters were given to the 162 midwives to participate in the study and were instructed to contact the researcher by electronic mail or phone. Fifty-two (32\%) of the 162 midwives responded to the recruitment letter and 21 (13\%) midwives signed a consent to be interviewed.

\section{Data Collection and Analysis}

The interview questions about knowledge of G6PD deficiency assessed the following topics: general knowledge, cause, inheritance pattern, diagnosis, medical management, sources of information, and genetic counseling awareness. The interview questions were subsequently validated (Table 1). The validation of interview questionnaire was

Table 1. Interview Questions

Knowledge pertains to the information about (1) disease causation, (2) mode of inheritance, (3) genetic predisposition of G6PD deficiency and (4) management.

1. What is G6PD deficiency?

2. What causes G6PD deficiency?

3. How do people get G6PD deficiency?

4. How to test for the presence G6PD deficiency?

5. How to manage G6PD deficiency?

6. What foods or medications must be avoided to prevent complications when you have G6PD deficiency?

7. Where did you get your information about G6PD deficiency?

Self-Perceived Role refers to the midwives' self-perceived role towards working with G6PD deficient patients and also with their effectiveness concerning the care they provide their patients.

1. Parents with children with G6PD deficiency should receive proper counseling.

2. Health workers should receive proper education about G6PD deficiency.

3. Counseling parents of a child with G6PD deficiency should be performed by midwives.

4. Counseling G6PD-deficient patient should be performed by a separate health care worker.

5. I should refer G6PD deficient patients to other health care workers for counseling.

6. Quality of life of patients with G6PD deficiency will improve with proper counseling.

7. I feel confident in counseling parents with children with G6PD deficiency.

8. I am satisfied with the information that I shared with the parents of children with G6PD deficiency.

9. I am confident that the information that I am sharing to the parents satisfies their need to understand G6PD deficiency.

Experience refers to the midwife's previous experience in counseling parents with a G6PD-deficient child.

1. How many patients are you seeing in a week?

2. How many times have you encountered conditions included in the NBS panel or other conditions with genetic element during the last year?

3. How many times have you delivered or followed-up on a child with G6PD deficiency?

4. What information do you want to know about G6PD deficiency that would help you explain to the parents about the disease?

5 . When the NBS result was released, how did you explain it to them?

6. How do you feel after giving the information to the parent?

7. How confident were you in communicating the information to the parents? What suggestions do you have for improving parental education of G6PD? 
conducted by pre-testing on a small group of 13 midwives with a Cronbachs Alpha score of 0.7065 .

One-to-one semi-structured interviews were conducted with the participants. An interview guide was used and interview questions were asked using the Tagalog language, which was the language of choice of the participants. All interviews with the participants were tape recorded and then transcribed by the research assistant and cross checked by the primary investigator to ensure the validity of transcripts. Follow-up questions were asked to reinforce or clarify verbalized answers.

The interview transcripts were checked against the audio tapes for accuracy of the data. Transcripts were uploaded in the Dedoose. The program is a web application for qualitative and mixed methods research and has been fieldtested and journal-proven by leading academic institutions worldwide. Transcripts were further cross-checked using the participant's data. Excerpts were labeled and coded as "Adequate Knowledge" or "Inadequate Knowledge". Each correct answer corresponded to one point. The highest possible score that could be obtained from all items of the knowledge portion was 6 points. "Adequate Knowledge" meant that at least 4 out of 6 knowledge questions were answered correctly. "Inadequate Knowledge" meant that less than 4 questions were answered correctly. Six (6) knowledge questions distinguished respondents as having "Adequate Knowledge" vs. "Inadequate Knowledge" since these were considered close ended questions in which specific answers can be extracted.

\section{RESULTS}

\section{Knowledge}

All respondents were female and the majority (81\%) finished a degree in B.S. Midwifery. Most of the participants have had at least 21 years of practice and $67 \%$ of them had undergone newborn screening training. Table 2 summarizes the sociodemographic characteristics of the participants.

\section{Knowledge about G6PD deficiency}

All the participants encountered confirmed G6PD patients in their respective areas of practice. The majority (86\%) had inadequate general knowledge of G6PD deficiency (Table 3). Prevalent misconceptions about the cause of G6PD deficiency include: 1) G6PD deficiency was due to lack of glucose, 2) anemia, and or 3) maternal exposure of the mother to chemicals or drugs during pregnancy. Some (10\%) participants had knowledge of the role of genetics in G6PD deficiency but were not able to explain accurately.

Some $(10 \%)$ of participants knew that there was a need to refer confirmed cases to their doctors and to advice the parents of the importance of follow-up care with the child's pediatrician. Majority (95\%) of the respondents had knowledge of the management of G6PD deficiency and correctly identified foods and medications that should be avoided by the patients with G6PD deficiency.
Table 2. Socio-Demographic Characteristics

\begin{tabular}{lr}
\multicolumn{1}{c}{ Characteristics } & Frequency (\%) \\
1. Age (in years), mean & $50 \pm 7.16$ (range: 41-61) \\
\hline 2. Sex & $0(0)$ \\
Male & $21(100)$ \\
Female & \\
\hline 3. Marital Status & $2(9.5)$ \\
Single & $17(81)$ \\
Married & $2(9.5)$ \\
Widow & \\
\hline 4. Educational Attainment & $17(81)$ \\
Midwifery (Diploma) & $4(19)$ \\
Bachelor's degree & $2(10)$ \\
\hline 5. Individual monthly income (in Php) & $11(52)$ \\
1,000 - 10,000 & $3(14)$ \\
11,000 - 20,000 & $5(24)$ \\
21,000 - 30,000 & \\
31,000 - 40,000 & \\
\hline 6. Years in Practice & $2(9.5)$ \\
\hline 7. Expanded Newborn Screening Training & $14(66.7)$ \\
With & $5(23.8)$ \\
Without & \\
Cannot recall & $14(66.7)$ \\
\hline 8. Newborn Screening Training & $2(9.5)$ \\
With & $5(23.8)$ \\
Without & $3(14.3)$ \\
Cannot recall & $5(23.8)$ \\
\hline 9. G6PD Seminar & \\
With & \\
Without & \\
Cannot recall & \\
\hline 10. Heel Prick Method Seminar & $5-39)$ \\
With & \\
Without & \\
Cannot recall & \\
\hline & \\
\hline
\end{tabular}

Table 3. Knowledge of G6PD Deficiency among 21 Midwives from Lipa and Manila

\section{Knowledge Areas}

Frequency (\%)

1. Knowledge about what is G6PD deficiency Adequate knowledge Inadequate knowledge

$3(14 \%)$ $18(86 \%)$

2. Knowledge about the cause of G6PD deficiency Adequate knowledge

$0(0 \%)$ Inadequate knowledge $21(100 \%)$

3. Knowledge about the Inheritance pattern of G6PD deficiency Adequate knowledge Inadequate knowledge

4. Knowledge about Diagnosis of G6PD deficiency Adequate knowledge $21(100 \%)$ Inadequate knowledge

5. Knowledge about the management of G6PD deficiency Adequate knowledge Inadequate knowledge $1(5 \%)$

6. What foods or medications must be avoided to prevent complications

Adequate knowledge Inadequate knowledge

* "Adequate Knowledge" meant that at least 4 out of 6 knowledge questions were answered correctly. "Inadequate Knowledge" meant that less than 4 questions were answered correctly. 
Since the participants were asked about their knowledge of the disease, it was important to recognize where in particular the midwives obtained their G6PDrelated information. Majority (76\%) of the participants said that they acquired their knowledge from seminars, $14 \%$ from doctors who reported the results to the parents, and $10 \%$ from materials provided by the Newborn Screening Program.

\section{Self-perceived role}

The participants were asked about their perceived role with regard to genetic information about patients with G6PD deficiency. When asked whether midwives should provide genetic counseling to parents, 91\% of Manila participants disagreed while $70 \%$ of participants from Lipa agreed. Majority of the participants from Manila (91\%) and Lipa (90\%) agreed that counseling G6PD deficiency should be performed by other health care worker other than midwives. All participants from Manila and Lipa agreed that parents with G6PD deficiency affected children should receive proper genetic counseling and health workers should receive proper education about G6PD deficiency. All Manila participants and only 80\% Lipa participants agreed that they should refer G6PD deficiency patients to other health care workers for counseling. All midwives in Manila and Lipa agreed on the following statements: (1) Quality of life of patients with G6PD deficiency will improve with proper genetic counseling, (2) they felt confident in counseling parents with children affected with G6PD deficiency, (3) they were satisfied with the information they shared with the parents of children affected with G6PD deficiency, (4) and they were confident that the information that they shared satisfied the parents' needs for understanding G6PD deficiency.

\section{Experience}

The participants expressed sympathy to parents of patients with G6PD deficiency. The midwives presented some of the emotional responses during their clinic encounter with the patient and the parents as they shared experiences and information with them. All participants expressed satisfaction with the information and care that they provided to the parents and patients. Generally, most participants reported that they were confident in the process of giving information whereas $17 \%$ were not. There were three (3) participants who did not provide any answer nor explanation on this topic. In comparison, all participants from Lipa were confident while $70 \%$ of participants from Manila were confident in giving G6PD deficiency-related information to parents and families.

One participant commented that she was confident in providing G6PD deficiency-related information but acknowledged her incomplete understanding of the disorder and need for additional information and training. Some (10\%) midwives expressed confidence in giving information at the same time referred the patient and their parents to the medical doctor and offered seminars or trainings as sources of additional information and support.

\section{Suggestions for G6PD education}

Majority of the participants suggested that education on G6PD deficiency should not be limited to healthcare workers but should also include parents and future parents.

Some (5\%) participants emphasized that lectures during prenatal check-ups of pregnant women should be a venue for giving information about the conditions in the Newborn Screening panel. The participants suggested distributing posters and information materials about the condition. One participant suggested the use of an identification card that reflects the medical status, medical and diet restrictions of the infant or child diagnosed with G6PD deficiency. One respondent suggested an improved information education and communication (IEC) materials with regard to G6PD that can be easily understood by the parents. In addition, the participants recommended that some trained personnel, such as doctors and nurses, with background on genetics should be available and should be the ones giving the information to the parents. Specific suggested tools for G6PD education can be seen on Table 4.

Table 4. Suggestions for G6PD Education

\begin{tabular}{lr}
\multicolumn{1}{c}{ Suggestions } & Frequency (\%) \\
\hline $\begin{array}{l}\text { Seminars and trainings to the parents and } \\
\quad \text { health workers }\end{array}$ & $15(71 \%)$ \\
TV advertisements & $2(10 \%)$ \\
Lectures during Prenatal clinics & $2(10 \%)$ \\
ID system to G6PD patients & $1(5 \%)$ \\
More detailed reading materials & $1(5 \%)$ \\
Trained personnel & $2(10 \%)$ \\
\hline
\end{tabular}

\section{DISCUSSION}

The present study revealed that all midwives from Lipa and Manila know how G6PD Deficiency is detected and 95\% had adequate knowledge on the management on foods and medications that should be avoided. This knowledge boosted their confidence in providing counseling to the families as well as educating them on the management of G6PD deficiency.

However, when assessed on the knowledge on the genetics aspect of G6PD deficiency, the study revealed that they had inadequate knowledge on the etiology of G6PD deficiency and its inheritance pattern. These findings were similar to the results from previous literature, i.e., midwives did not have enough information on basic genetic topics, human genetic diseases, and prenatal diagnosis of genetic diseases, genetic screening, and genetic counseling. ${ }^{8,9,10}$ The lack of knowledge was evident since no explanation was made on the scientific/genetic cause of G6PD deficiency in the family's past pregnancies. 
The inability of the parents to fully understand G6PD deficiency may result to less understanding of the recurrence of the condition in the future pregnancies and eventually non-compliance to the recommended NBS guidelines on dietary management, dietary restrictions and follow-up. This could be prevented by providing information about the inheritance pattern of G6PD deficiency, emphasizing the chance of recurrence of the condition in future pregnancies and providing complete and accurate information on the management of G6PD deficiency.

As highlighted in the results, $91 \%$ of the participants in Manila area (urban setting) disagreed with the statement that they should perform genetic counseling to parents of patients with G6PD deficiency. Reasons of the respondents' response can be explained with their lack of complete knowledge on the etiology and inheritance of G6PD deficiency and their preference to practice their midwifery profession instead of a new field of genetic counseling. Furthermore, the participants could easily refer their patients to the Philippine General Hospital, the largest tertiary hospital in the country where they have access to genetic counseling services. While $70 \%$ of participants from Lipa (rural setting) agreed that they should perform the counseling since it was observed that they were the only accessible resource person who could provide health education in the said area. This activity enabled the participants from the rural area to exercise their skills in counseling and giving information thereby boosting their confidence in taking the task as the parents' educator and counselor.

\section{The Midwife as the primary health educator}

All participants reported that the parents with children diagnosed with G6PD deficiency should receive proper counseling and that healthcare workers should receive proper education about G6PD deficiency. All participants felt confident on the aspect of giving their onhand knowledge on the condition to the parents that they gained through training and seminars. In contrast with the results of the study of Benjamin et al., wherein only 11$17 \%$ of the participants felt confident or very confident in carrying out procedures/skills that may require genetic knowledge. ${ }^{11}$ This may be a result of the participants' experience in their everyday exposure to their patients, in which they were considered as highly knowledgeable.

\section{Midwife's experience/encounter with parents of patients who are confirmed cases for G6PD deficiency}

All participants showed confidence in giving information about G6PD deficiency to the parents. This exhibits their eagerness to reach out and provide health service to their patients. Even though the participants displayed enthusiasm, they still preferred an expert to provide health education and counseling. Referral of patients to other health care experts for counseling would also mean more time for them to carry out other important tasks.

Apart from the traditional source of information to educate parents about G6PD deficiency, the participants also suggested using television in the form of advertisements from major television companies in the country as one alternative source of information. This way, information can be communicated to parents with or without children with G6PD deficiency and also to all health care workers. This may bring positive outcomes, however it may also provide confusion and anxiety brought by unfamiliar medical jargon if the information is not in layman's terms.

In general, it was observed that not all participants have the capacity to provide all technical information on G6PD deficiency to the parents of the patients. The participants lack understanding of the condition but were able to provide information on the management of G6PD deficiency. Many of the participants still recommended that there should be an expert to provide the necessary information during counseling.

\section{Study Limitations}

G6PD-deficient patients and families acquire care from different health care providers including physicians, nurses, midwives, dieticians and social workers. This study only obtained information from two locations in the Philippines; the findings may not reflect knowledge, self-perceived role and experiences in other locations.

The primary method of data collection was a semistructured interview. Since this method relies on selfreported data, potential sources of bias include selective memory (remembering or not remembering experiences or events that occurred at some point in the past), and exaggeration. The possible challenge of the study participants in expressing themselves and hesitation in reporting negative issues they encountered may be a source of bias.

The study only focused on two areas - Manila City and Lipa, Batangas. Hence, the study did not attempt to generalize its results to the rest of the regions of the country.

\section{Recommendations and Conclusion}

The results of the study may help improve G6PD deficiency education seminars, information dissemination efforts, and policies for midwives and other health care providers accountable for counseling parents of children with G6PD. Midwives should be made aware of the G6PD quarterly seminars conducted by the Newborn Screening Reference Center (NSRC), so they may participate. Furthermore, annual recertification of professionals like midwives should include trainings and seminars that focus on the enhancement of their knowledge specifically on common genetic conditions such as G6PD deficiency. The NSRC, which provides information and resources to health professionals on the area of newborn screening, is suggested to review and improve teaching guidelines regarding 
newborn screening to midwifery students. Information, education, and communication materials such as brochures and pamphlets should contain information on basic genetics and the inheritance pattern of the condition. These resources would help midwives educate parents with children of such conditions and future parents as well. Given that midwives provide inadequate information to parents with G6PD deficiency patients, there should be available genetic experts in rural areas to provide accurate information and to ensure management compliance and continuity of care.

Since the study only focused on the knowledge, selfperceived role, and experiences of the midwives from Manila and Lipa, it is still recommended that a thorough investigation be considered in other areas of the country such as in the Visayas and in Mindanao. Other health care providers such as nurses and doctors should also be considered when investigating the process of giving health education and counseling to patients.

In conclusion, with limited genetic counseling professionals available in the country, midwives play a vital role in providing sufficient and accurate information regarding genetic conditions such as G6PD deficiency in urban and in community settings. They are deployed in every health center all over the country, serving as the main resource person of families and patients with genetic condition. Utilizing the skills and proximity of our midwives in providing complete and accurate information regarding common genetic conditions is highly recommended. In order to address knowledge gaps of health care professionals, proper education from training institutions should be done, with emphasis on the genetic aspect of most common genetic conditions in the country. Health care facilities in rural and urban areas must participate in educating their health care officers regarding the genetic aspect of most common genetic conditions in the country for them to cater information needs of families affected with genetic conditions. In the long run, the expertise of genetic counselors should be accessible to every Filipino patient and their families.

\section{Acknowledgments}

Acknowledgments go to all midwives who participated in the study. The authors also sincerely thank Dr. MaAm Joy Tumulak and Mrs. Cindy Grace Guerbo for their critical comments and editing.

\section{Statement of Authorship}

All authors have approved the final version submitted.

\section{Author Disclosure}

All authors declared no conflicts of interest.

\section{Funding Source}

This study received a grant from the Newborn Screening Reference Center National Institutes of Health University of the Philippines Manila (Project Code: NSRC 2015-008).

\section{REFERENCES}

1. Newborn Screening Reference Center. Prevalence of Disorder among Filipino Newborns (1996-2016) [Internet]. [cited 2017 Feb 7]. Available from: http://www.newbornscreening.ph.

2. Mason PJ, Bautista JM, Gilsanz F. G6PD deficiency: the genotypephenotype association. Blood Rev. 2007 Sep; 21(5):267-83.

3. Singh H. Glucose-6-phosphate dehydrogenase deficiency: a preventable cause of mental retardation. Br Med J. 1986 Feb; 292(6517):397-8.

4. Tseng CP, Huang CL, Chong KY, Hung IJ, Chiu DTY. Rapid detection of glucose 6 phosphate dehydrogenase gene mutations by denaturing high-performance liquid chromatography. Clin Biochem. 2005 Nov; 38(11):973-80. doi: 10.1016/j.clinbiochem.2005.07.015.

5. Leong A. Is there a need for neonatal screening of glucose-6phosphate dehydrogenase deficiency in Canada? McGill J Med. 2007 Jan; 10(1):31-4.

6. DOH Circular No.333. Rules and Regulations Implementing Republic Act No. 9288 otherwise known as the "Newborn Screening Act of 2004". 2004.

7. Laurino M, Padilla CD, Alcausin MM, Silao CL, Cutiongco-de la Paz EM. A Master of Science in Genetic Counseling Program in the Philippines. Acta Med Philipp. 2011; 45(4):7-9.

8. De D. How well does midwifery education enable professionals to work with families and individuals affected by sickle cell and thalassemia? Research Policy and Planning. 2006; 24(2):121-33.

9. Tomatır AG, Ozsahin A, Sorkun HC, Demirhan H, Akdag B. Midwives' approach to genetic diseases and genetic counseling in Denizli, Turkey. J Genet Couns. 2006 Jun; 15(3):191-8.

10. Adebami OJ. Assessment of knowledge on causes and care of neonatal jaundice at the Nigerian primary and secondary health institutions. Int J Res Med Sci. 2015 Oct; 3(10):2605-12.

11. Benjamin CM, Anionwu EN, Kristoffersson U, ten Kate LP, Plass AMC, Nippert I, et al. Educational priorities and current involvement in genetic practice: a survey of midwives in the Netherlands, UK and Sweden. Midwifery. 2009 Oct; 25(5):483-99. doi:10.1016/j.midw.2007.08.003 